\title{
Profiling and modeling of dc nitrogen microplasmas
}

\author{
Chester G. Wilson ${ }^{\mathrm{a})}$ and Yogesh B. Gianchandani \\ EECS Department, University of Michigan, Ann Arbor, Michigan and Department of Electrical \\ and Computer Engineering, University of Wisconsin, Madison, Wisconsin
}

Robert R. Arslanbekov and Vladimir Kolobov

CFD Research Corp., Huntsville, Alabama

Amy E. Wendt

Department of Electrical and Computer Engineering, University of Wisconsin, Madison, Wisconsin

(Received 26 February 2003; accepted 5 June 2003)

\begin{abstract}
This article explores electric current and field distributions in dc microplasmas, which have distinctive characteristics that are not evident at larger dimensions. These microplasmas, which are powered by coplanar thin-film metal electrodes with $400-\mu \mathrm{m}$ minimum separations on a glass substrate, are potentially useful for microsystems in both sensing and microfabrication contexts. Experiments in $\mathrm{N}_{2}$ ambient show that electron current favors electrode separations of $4 \mathrm{~mm}$ at 1.2 Torr, reducing to $0.4 \mathrm{~mm}$ at 10 Torr. The glow region is confined directly above the cathode, and within $200-500 \mu \mathrm{m}$ of its lateral edge. Voltage gradients of $100 \mathrm{kV} / \mathrm{m}$ exist in this glow region at 1.2 Torr, increasing to $500 \mathrm{kV} / \mathrm{m}$ at 6 Torr, far in excess of those observed in larger plasmas. Numerical simulations indicate that the microplasmas are highly nonquasineutral, with a large ion density proximate to the cathode, responsible for a dense space-charge region, and the strong electric fields in the glow region. It is responsible for the bulk of the ionization and has a bimodal electron energy distribution function, with a local peak at $420 \mathrm{eV}$. (C) 2003 American Institute of Physics. [DOI: 10.1063/1.1595143]
\end{abstract}

\section{INTRODUCTION}

On-chip microplasmas and microdischarges have been shown to have potential applications for localized silicon etching, ${ }^{1}$ for spectroscopy of water impurities, as a potential tunable UV source, ${ }^{2,3}$ for gas spectroscopy, ${ }^{4,5}$ and for display technology. ${ }^{6}$ All of these potential applications rely on the localization of the microplasmas or microdischarges. This is typically accomplished by physical confinement within a microcavity, but self-confinement can also be accomplished. Small-scale dc electric discharges that operate in the 1-20Torr pressure regime sustain a glow region that self-localizes over the cathode. ${ }^{7,8}$ This glow region supports large voltage gradients and is by far the most optically intense region of the discharge. (Modeling results presented here suggest that this region provides the bulk of the plasma ionization.)

While some previous large-scale dc plasma experiments and modeling efforts show trends similar to those for the microplasmas just described, the physics of the glow localization on either miniaturized or coplanar electrodes remains unexplored. Several self-consistent fluid models have been used to calculate the properties of dc glow discharges, coupling solutions to the Boltzman and Poisson equations. This technique was used to correlate the cathode-fall voltage and distance to the current density in dc discharges in rare, and some molecular, gases. ${ }^{9}$ Similar efforts, including continuity of momentum equations, were used to analyze $\mathrm{CO}_{2} \cdot{ }^{10} \mathrm{~A}$ continuum model for the entire plasma region of rf and dc discharges was developed, for pressure ranges of 0.5 to 5 Torr, an assumed Maxwell-Boltzmann electron energy dis-

${ }^{\text {a)} E l e c t r o n i c ~ m a i l: ~ c h w i l s n @ e n g i n . m i c h . e d u ~}$ tribution, and a parallel-plate configuration, where the plate diameter was large compared to the spacing, to allow a onedimensional (1D) solution. ${ }^{11}$ These efforts were extended to account for fast, nonequilibrium electrons in the cathode-fall region. ${ }^{12}$ The modeling effort in Ref. 12 found that the largest ionization rates are in the negative glow region proximate to the cathode fall, and that the ionization rate, and the region of optical intensity got close to the cathode as the pressure was increased to 2 Torr. Similar results for ionization and optical intensity of $\mathrm{H}_{2}$ dc glow discharges at 20-30 Torr were found, with a $1 \mathrm{D}$ solution. ${ }^{13}$

Kinetic models have also been successful in modeling dc glow discharges. A two-dimensional (2D) self-consistent kinetic model coupling the Boltzmann and Poisson equations was developed, and used to study the transition between normal and abnormal glow regimes. ${ }^{14}$ The cathode-fall and negative glow regions of dc plasmas have been investigated experimentally and theoretically, again for large-scale parallel-plate plasma discharges. A kinetic model using the Monte-Carlo technique was used to calculate the ionelectron current balance in the sheath region of a He dc parallel plate discharge. ${ }^{15} \mathrm{~A}$ single-beam kinetic model with two varying parameters of density and velocity for electrons was developed to analyze the cathode fall, ${ }^{16}$ as well as a selfconsistent kinetic model. ${ }^{17}$

In contrast to past efforts, this article focuses on characterizing and modeling dc microplasmas generated from coplanar electrodes patterned from thin film electrodes on a glass microchip. Optimizing localized optical intensity, plasma densities, and electron energies are essential to the design of micro electro mechanical-systems related sensing and manufacturing applications. Experimental results charac- 


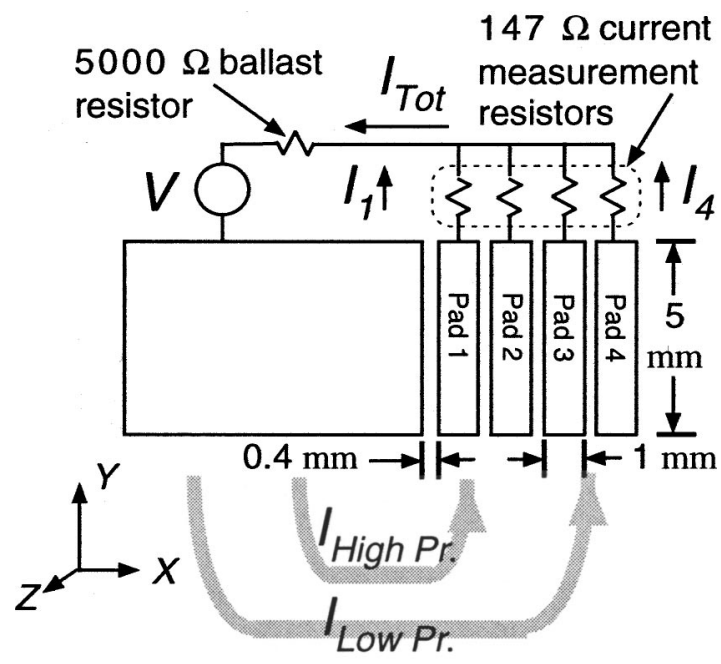

FIG. 1. Planar Ti electrode configuration, patterned on glass to measure cathode current density. Cathode is split into separate paths, where the current is independently measured. Polarity can be reversed to measure anode current density.

terizing these microplasmas are presented in the next section, followed by modeling efforts in Sec. IV.

\section{EXPERIMENTAL RESULTS}

In order to measure the spatial distribution characteristics of the anode and the cathode current, an $\mathrm{N}_{2}$ microplasma was created between planar thin-film Ti electrodes patterned on a glass substrate at vacuum levels ranging from 1 to 20 Torr. A segmented electrode $1 \mathrm{~mm}$ wide, $5 \mathrm{~mm}$ long, and spaced $400 \mu \mathrm{m}$ apart was used first as an anode, and then as a cathode to measure these characteristics, which are dependent on both pressure and applied voltage (Fig. 1). Pads 1 through 4, varying only in position along the $X$ axis were connected through separate $147-\Omega$ resistors, so that the current traveling through them could be independently measured. A $5000-\Omega$ ballast resistor was used to limit the total plasma current. The current distribution in the anode and cathode is described in Sec. II A, and the internal voltage structure of the glow region of microplasmas is described in Sec. II B.

\section{A. Current distribution}

Figure 2 shows the current distribution as a function of applied power for 2 Torr. The cathode current is nearly uniform through all electrodes for all applied voltages. For the case of the anode current, the electrode of maximum current becomes pad 2, with $45 \%$ of the current at the highest voltage, and the minimum current electrode is pad 4 , with $15 \%$. The current in the innermost and the outermost electrodes, as a percent of total current versus pressure, is shown in Fig. 3. These data are for the anode and the cathode current distributions, all at $400 \mathrm{~V}$. In the case of the cathode current, at 1.2 Torr, the current is marginally higher in the outermost electrode. At higher pressures, the current becomes highest in the innermost electrode, with $34 \%$ of the current going to the inner electrode, and $18 \%$ in the outer electrode for the case

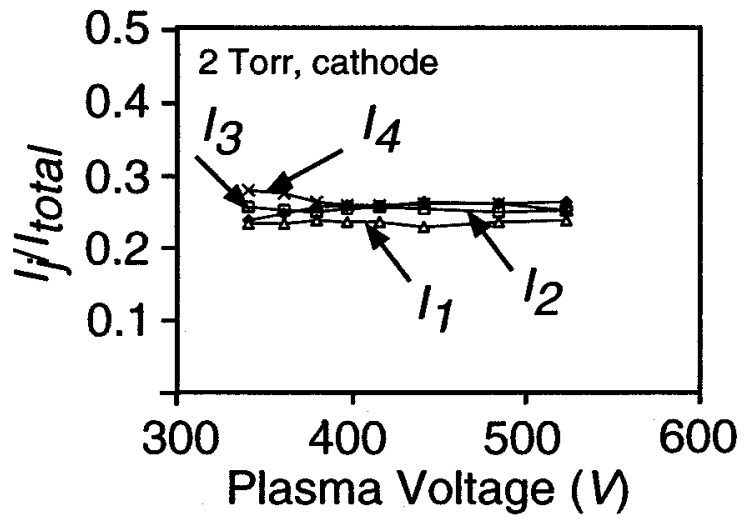

(a)

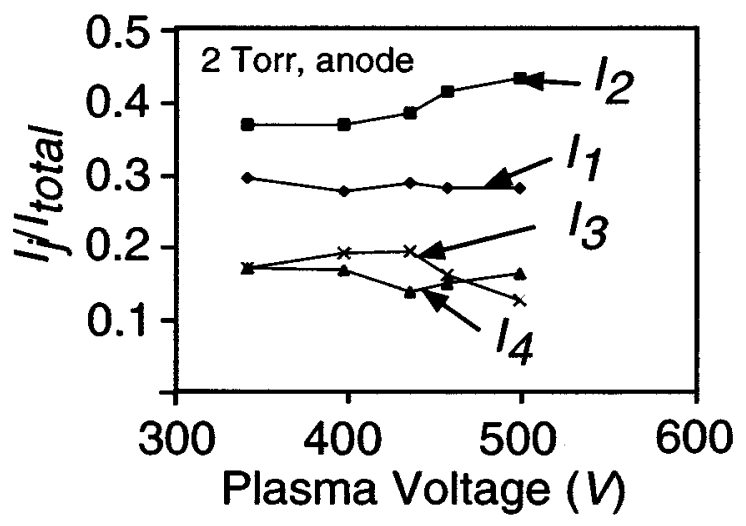

(b)

FIG. 2. Current reconstruction for 2 Torr $\mathrm{N}_{2}$ : (a) cathode (b) anode.

of 10 Torr. The effect is much greater for the anode current. At 10 Torr, essentially all of the current goes to the closest electrode.

This asymmetry between anode and cathode current is believed to be caused in part by the difference in mobilities between electrons, which form the anode current and are more easily deflected by the electric field, and positive ions, which form a significant component of the cathode current and are heavier. A portion of the cathode current is also

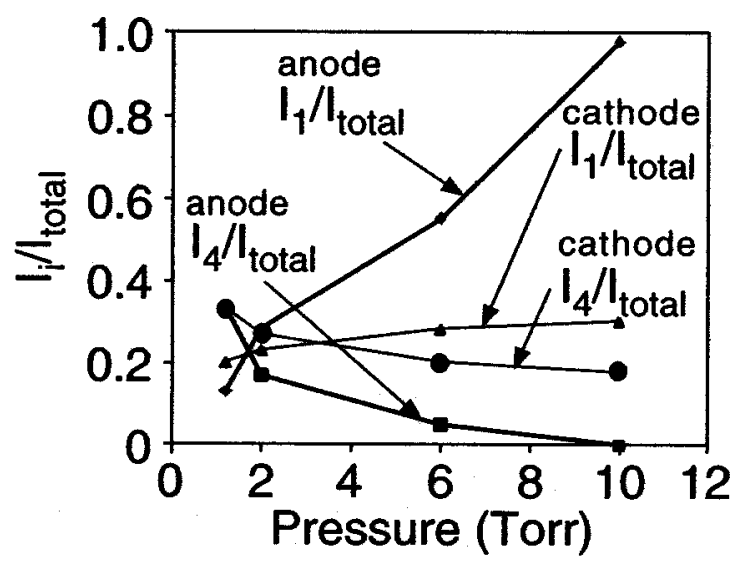

FIG. 3. Anode and cathode current distribution for pressures ranging from 1.2 to 10 Torr. 


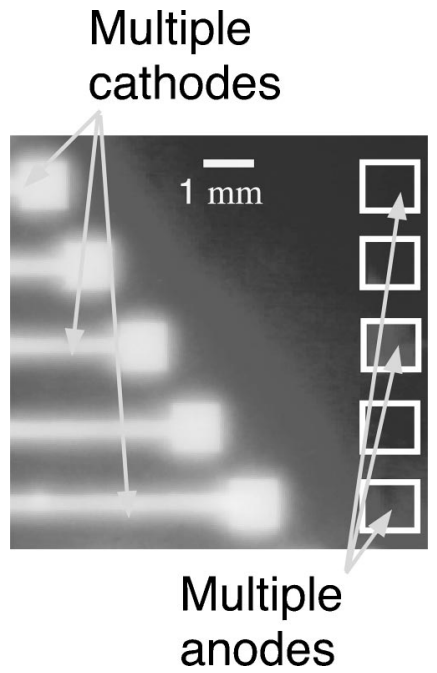

FIG. 4. Microplasmas generated at 2 Torr, in air, at $423 \mathrm{~V}$. The microplasma glow region is confined over the cathode, and is independent of the anodecathode spacing.

formed by secondary electrons, which are emitted where the ions strike the cathode. The spatial extent of the secondary electrons is, therefore, largely governed by the trajectory of the heavier ions. Secondary electrons also have a significant role in the powering and plasma generation of dc microplasmas as explained subsequently. These electrons are believed to cause a large amount of ionization proximate to the cathode, which also serves to make the current over this electrode more uniform.

When a microplasma is generated between two coplanar electrodes, the glow is confined to the region directly over the cathode. This can be seen in Fig. 4, which is a microplasma generated in air at 2 Torr, between independent 1.2$\mu \mathrm{m}$-thick Ti electrodes patterned on a glass substrate. The glow region is confined to the area located directly over the cathode, and the confinement to this region is independent of the anode-cathode spacing. The confinement is seen to be a function only of the pressure and the power density. As the pressure is increased, the glow is more closely confined, as power is increased, the plasma is more poorly confined. The localization of the glow over the cathode suggests that the ionization events in microplasmas are largely dependent upon secondary electrons, which are emitted by the cathodes and are accelerated by the electric field into the region above.

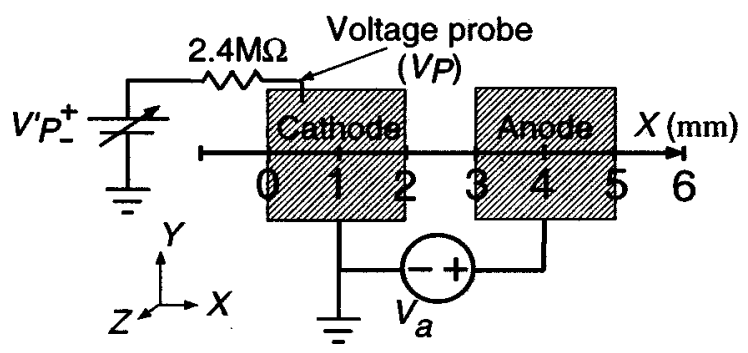

FIG. 5. Setup for the floating potential characterization experiment. Measurement circuitry for floating potential, and electrode geometry.

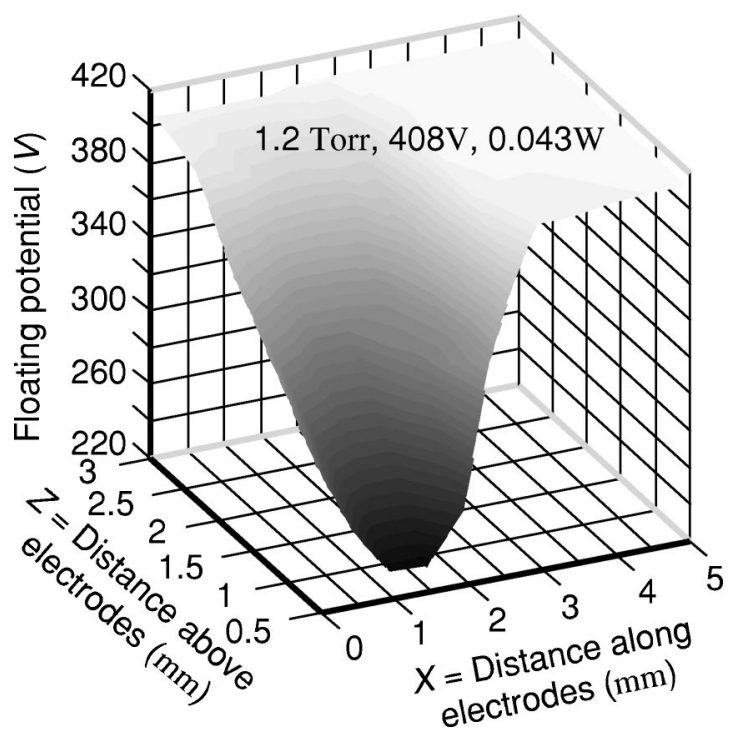

FIG. 6. Floating potential of an $\mathrm{N}_{2}$ microplasma at 1.2 Torr.

\section{B. Floating potential profile}

Measurements of the potential distribution in microplasmas were performed using passive diagnostic probes, as seen in Fig. 5. A microplasma was generated between the anodecathode pair, which was again formed by Ti patterned on a glass wafer. The electrodes were $2 \mathrm{~mm} \times 2 \mathrm{~mm}$, with a 1 -mm electrode spacing. The discharge was driven by a dc voltage source $V_{a}$. The voltage probe was inserted into the region above the anode and the cathode, and was scanned along the out-of-plane $Z$ axis, or the $X$ axis defining electrode spacing. At each point, the local floating potential $V_{P}$ was found by varying $V_{P}^{\prime}$ until the current through the resistor is reduced to zero. The voltage probe was constructed from a solid copper wire with a cross section of $0.013 \mathrm{~mm}^{2}$. The probe was coated with Teflon ${ }^{\mathrm{TM}}$ insulation so that the exposed end formed only a circular metallic cross section. When the electrodes were energized, the glow region is seen to be confined directly above the cathode. The microplasmas floating potential was mapped as a function of pressure and power density. In all reported cases, the plasma current did not vary by more than $2 \%$ throughout the duration of the floating potential measurements; therefore, it can be assumed the probe did not significantly perturb the microplasma.

Figure 6 shows a three-dimensional (3D) plot of the floating potential for a microplasmas generated at 1.2 Torr. Figure 7 illustrates contour plots for the floating potential profiles for microplasmas generated at 1.2 Torr at two different operating voltages. The figures map the $V_{P}$ in the space above the two electrodes. Three observations of microplasmas can be made on the basis of these results. First, there is no discernable voltage drop over the anode, the only significant electric fields exist directly over the cathode. Second, there is a considerable voltage gradient in the glow region of microplasmas. Third, in viewing the floating potential profiles for 1.2 Torr, the voltage gradient increases near the cathode and decreases more near the top of the discharge for the case of the higher operating voltage: the profile appears more like a traditional plasma. 

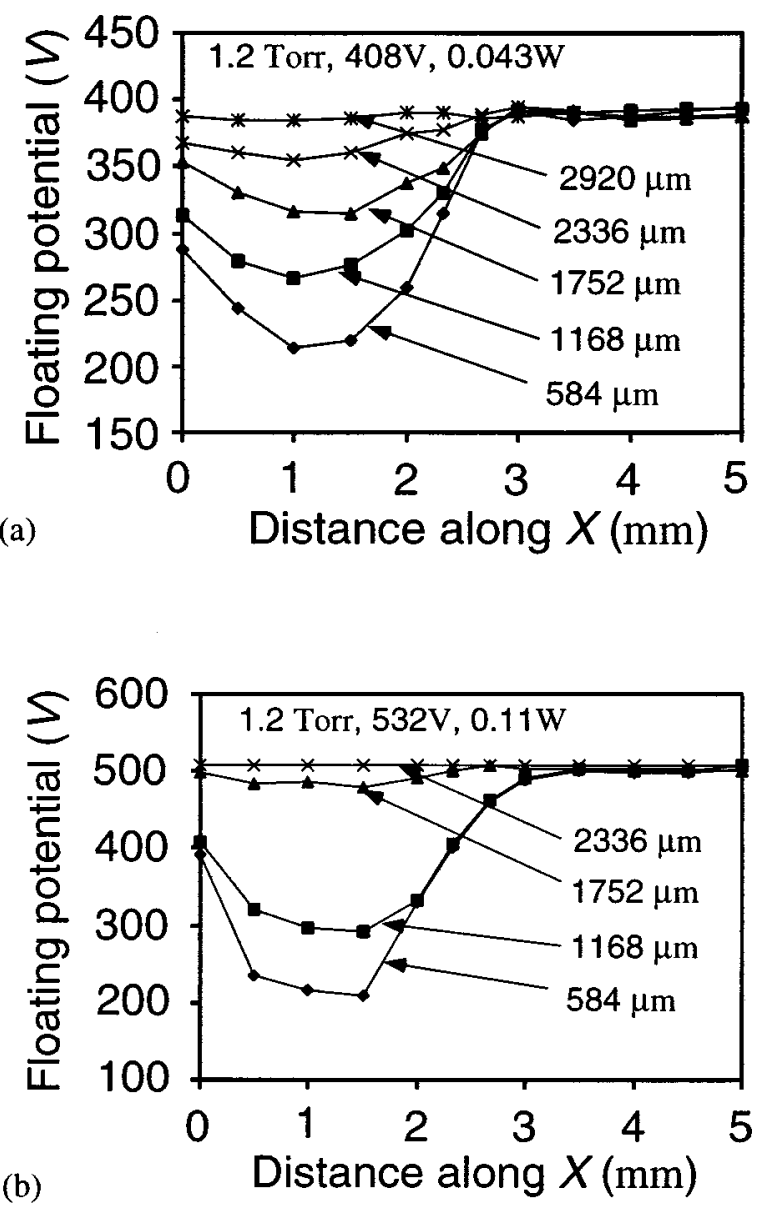

FIG. 7. Floating potential measurements for 1.2 Torr plasma operation at (a) $421 \mathrm{~V}, 0.045 \mathrm{~W}$ and (b) $532 \mathrm{~V}, 0.11 \mathrm{~W}$.

Figure 8 further illustrates the voltage gradient along the $Z$ axis (perpendicular to the plane of the wafer) at $X$ $=1.2 \mathrm{~mm}$ for the electrode configuration shown in Fig. 5. As the pressure is increased, the extent of the glow decreases and the voltage gradient increases. Voltage gradients range from 100000 to $500000 \mathrm{~V} / \mathrm{m}$.

In order to demonstrate the insensitivity of the field and glow region to electrode separation, an arrangement identical to that in Fig. 5-except that the electrode spacing was quadrupled to $4 \mathrm{~mm}$ - was used to generate a microplasma at the same pressure and power density. The resulting floating potential profile, shown in Fig. 9, is essentially identical to Fig. 6(a).

\section{MODELING}

The experimental results were reproduced and illuminated by computational modeling. Details of the model can be found in Ref. 17. Briefly, the plasma module solves continuum equations for electrons and ions coupled to Poisson's equation for the electrostatic field. The plasma module is self-consistently coupled with the kinetic module in Ref. 18, which solves a four-dimensional (three dimensions in space and one dimension in energy) electron Boltzmann equation for the electron distribution function (EDF). The chemistry module in Ref. 18 allows modeling of various plasma species. The plasma species included in the model, among oth-

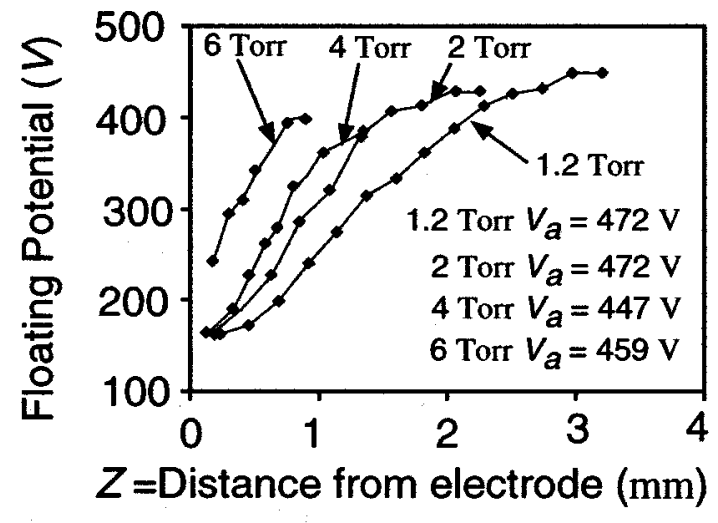

FIG. 8. Floating potential of $\mathrm{N}_{2}$ microdischarge at varying pressures, as a function of $Z$, distance above cathode at $X=1.2 \mathrm{~mm}$. Glow height decreases and voltage gradient increases with increasing pressure.

ers, were two metastable and two resonant $\mathrm{N}_{2}$ states, and molecular ions, with 10 species in total: $\mathrm{N}_{2}, \mathrm{~N}, \mathrm{~N}_{2}\left(\mathrm{~A}^{3} \Sigma_{u}^{+}\right)$, $\mathrm{N}_{2}\left(\mathrm{~A}^{3} \Sigma_{u}^{-}\right), \mathrm{N}_{2}\left(\mathrm{C}^{3} \Pi_{u}\right), \mathrm{N}_{2}\left(\mathrm{~B}^{3} \Pi_{g}\right), \mathrm{N}^{+}, \mathrm{N}_{2}^{+}, \mathrm{N}_{3}^{+}$, and $\mathrm{N}_{4}^{+}$. The presence of molecular ions was taken into account since the gas pressure can be relatively high under the studied conditions ( $>2-4$ Torr). The set of chemical reactions for $\mathrm{N}_{2}$ (Ref. 19) included various ionization and excitation steps, including stepwise ionization from metastables states, associative ionization, and other processes. The electron-induced reaction rates were calculated based on electron temperature, which was based on the solution of the Boltzmann equation for the EDF. The discharge was assumed to be maintained by secondary electron emission from the cathode surface due to ion bombardment (with a secondary electron coefficient of $0.01)$.

The voltage profile for microplasmas in $\mathrm{N}_{2}$ was modeled for 1.2 and 4 Torr, and the equivalent contour plots are shown in Fig. 10. The simulations of Fig. 10 predict that the only appreciable voltage drop is in the region immediately over the cathode, which is in agreement with the measurements from Figs. 6-7. The floating potential along $Z$ was modeled for 1.2, 4, and 6 Torr, and is shown in Fig. 11. This model also closely resembles experimental data from Fig. 8.

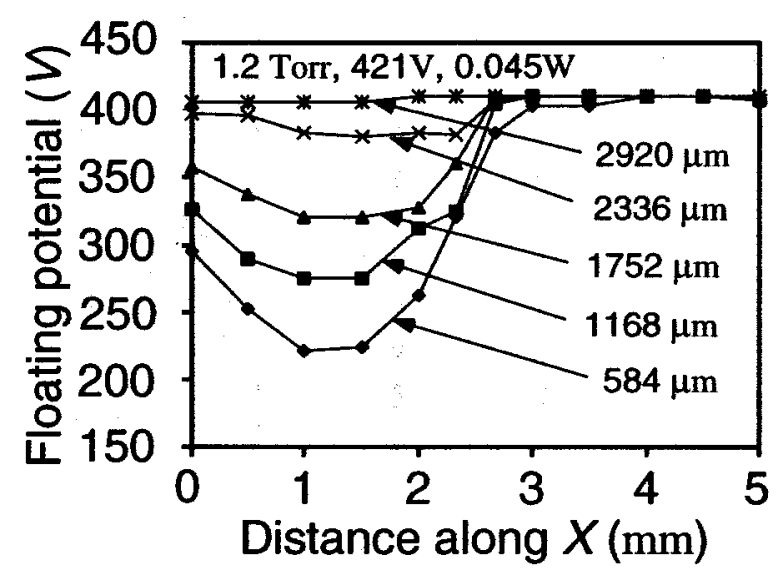

FIG. 9. Floating potential profile generated at 1.2 Torr, with similar power density and electrode configuration to that shown in Fig. 8, except that electrode spacing is $4 \mathrm{~mm}$, which is $4 \times$ greater. 

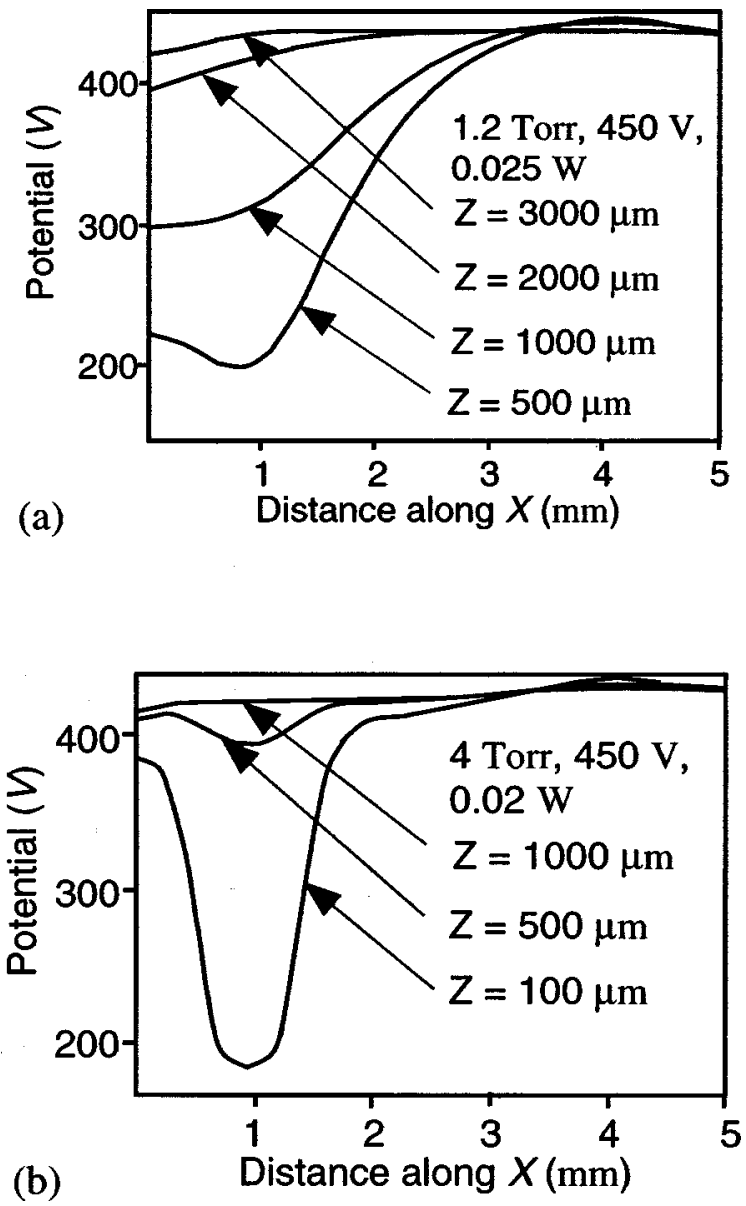

FIG. 10. Modeled floating potential profile contours generated at 1.2 Torr (a) and 4 Torr (b).

The simulation illustrates the increasing voltage gradient in the region of the cathode with pressure. The voltage drop becomes steeper as the electron neutral collisional cross section increases with pressure, causing the region of heavy ionization to decrease. Both the simulations and experimental measurements show average voltage gradients of around $300000 \mathrm{~V} / \mathrm{m}$ and $400000 \mathrm{~V} / \mathrm{m}$ for the 4- and 6-Torr cases, respectively.

The steep voltage gradients evident in microplasma discharges are a result of an ion space-charge region directly

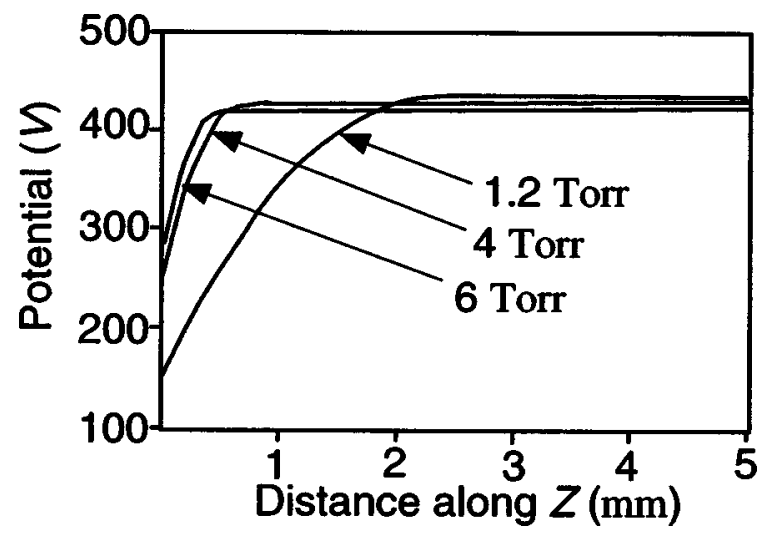

FIG. 11. Modeled floating potential profile along $Y$ for 1.2, 4, and 6 Torr.

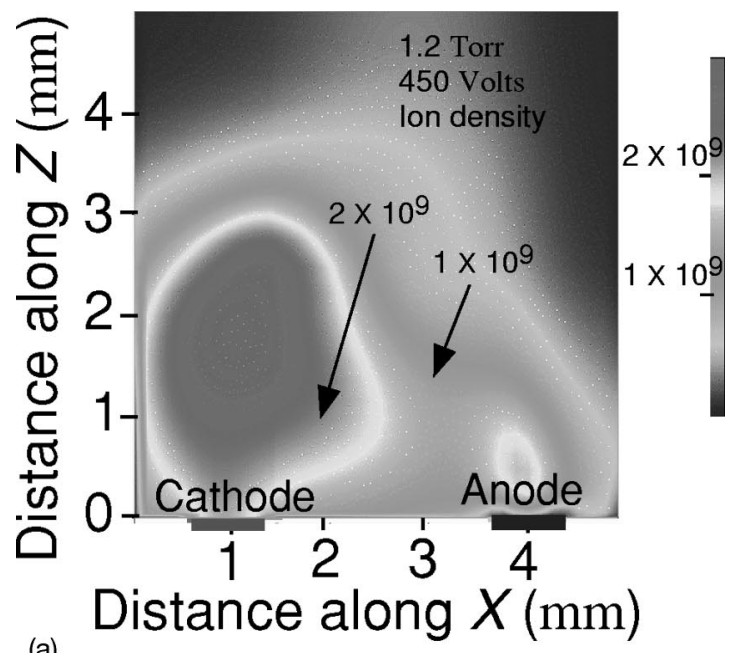

(a)

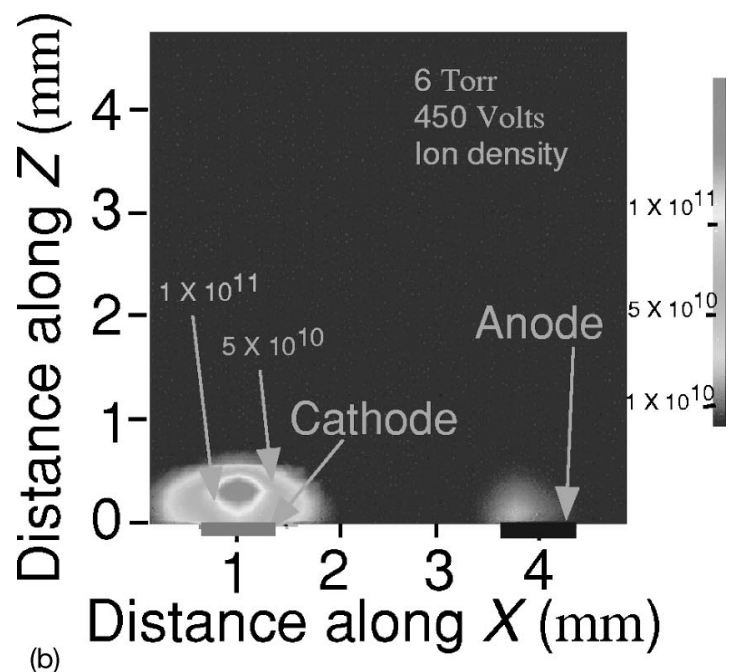

FIG. 12. Modeled ion density $\left(\mathrm{n} / \mathrm{cm}^{3}\right)$ for $\mathrm{N}_{2}$ microplasmas at 1.2 Torr (a) and 6 Torr (b).

above the cathode. The simulated ion density is shown in Fig. 12 for the 1.2 and 6 Torr cases. For this effort, the fluid model for both ions and electrons was used, assuming driftdiffusion motion for both species with a Maxwellian distribution of electrons. It can be seen that with increasing pressures, the ion density is confined closer to the region over the cathode. Less ionization occurs as the distance from the cathode is increased, and as collisional energy loss deteriorates the field-driven electron energy profile. This can be seen in the EDF in Fig. 13. The 3D EDF (2D in space and 1D in energy) was calculated using the fluid model parameters. The EDF is highly non-Maxwellian for nonthermal energies, containing a bimodal structure. (At thermal energies of up to several electron temperatures, the EDF is close to Maxwellian in the low-field region of the discharge, outside of the cathode-fall region.) The high-energy spike of this structure is due to the field-driven electrons, there is a sizable proportion of electrons with energy in excess of $400 \mathrm{eV}$ in the region proximate to the cathode. This attenuates in the region close to the anode, ceasing to support ionization. The electron density, shown in Fig. 14 is also highest in the over the cathode. In addition, it is symmetrical about the cathode at 

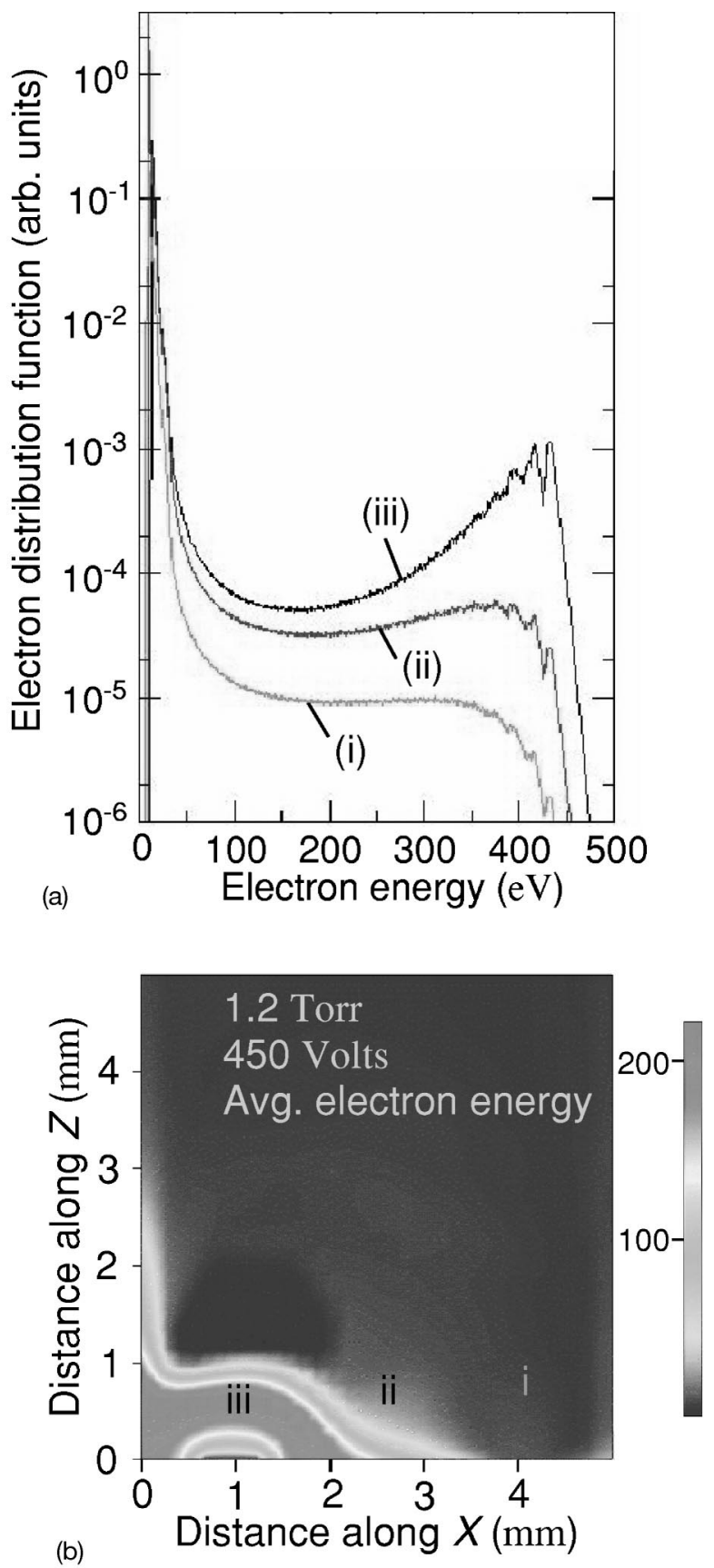

FIG. 13. Modeled electron energy distribution function has bimodal nature near the cathode.

higher pressures. This symmetry is not influenced by the location of the anode, as the trajectory of the electrons is dictated by the ion space-charge region. Due to the high electric fields that contribute to high velocities of charged species, substantial current can exist at lower particle densities. The electron density in the range of $10^{9}$ to $10^{11} / \mathrm{cm}^{3}$, which is lower than in conventional plasmas. The ion density is found to increase with pressure, a result of a greater number of electron-neutral collisions at higher pressures in the proximity of the cathode.

\section{CONCLUSIONS}

In conclusion, these results demonstrate how microscale dc plasmas vary considerably in breakdown and generation
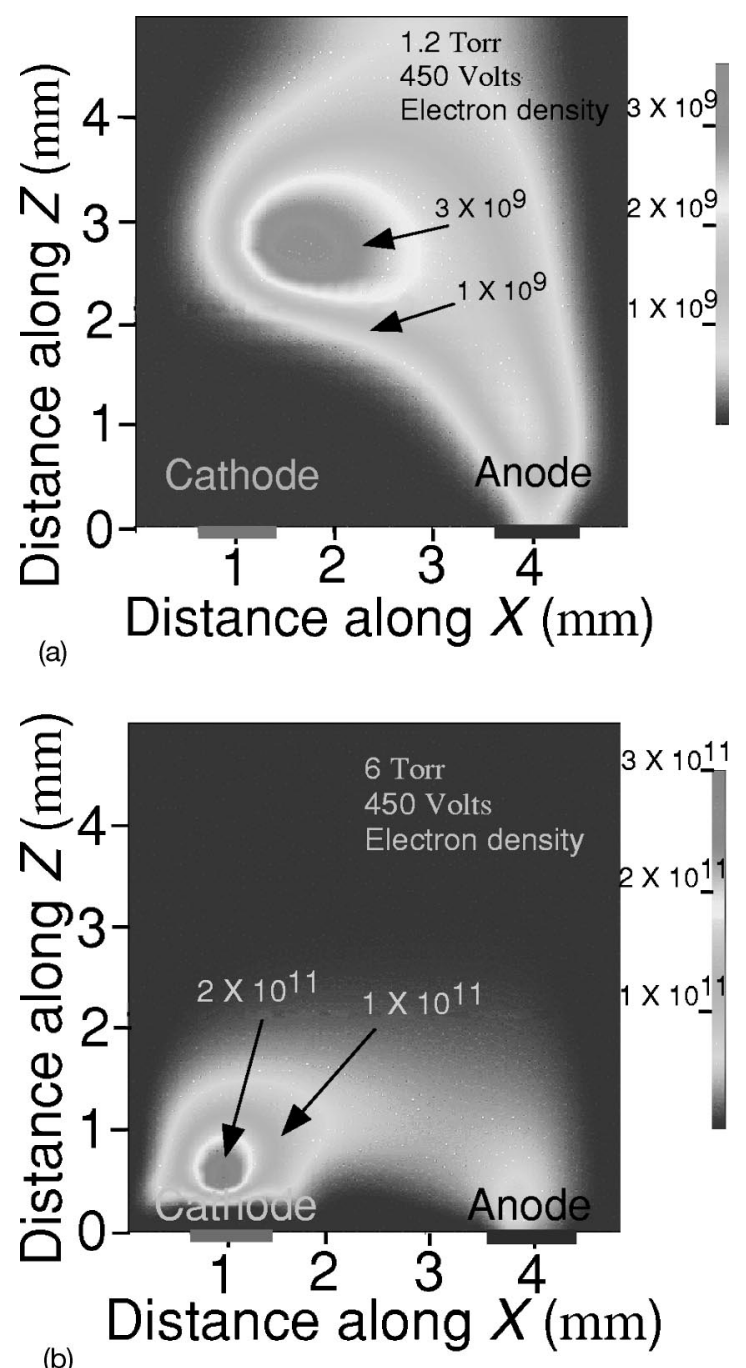

FIG. 14. Modeled electron density $\left(\mathrm{n} / \mathrm{cm}^{3}\right)$ for $\mathrm{N}_{2}$ microplasmas at 1.2 Torr (a) and 6 Torr (b).

parameters over macroscale plasmas. Most of the glow in microplasmas is confined directly over the cathode. This glow region supports a strong voltage gradient, in contrast to traditional plasmas. The localization of the glow to the cathode region is a result of field-driven secondary-emission electrons producing the bulk of the ionization in microplasmas. This results in a much higher electron energy distribution in the region proximate to the cathode, and correspondingly low electron densities. As the field-driven electrons become the dominant source of ionization, the space-charge region of ion density defines where plasma generation exists. The experimental effort was supported by modeling which showed generally good agreement. Modeling efforts show the discharges to be highly nonquasineutral, with a strong voltage gradient near the cathode, driving the bulk of the discharge ionization.

\section{ACKNOWLEDGMENTS}

This effort is supported in part by the National Science Foundation, US Geological Survey, and the Sea Grant. The authors thank Irena Gershovick for initial Monte-Carlo modeling. 
${ }^{1}$ C. Wilson and Y. Gianchandani, J. Microelectromech. Syst. 10, 50 (2001). ${ }^{2} \mathrm{C}$. Wilson and Y. Gianchandani, IEEE International Conference On Micro Electro Mechanical Systems, 2002, p. 248.

${ }^{3}$ G. Jenkins and A. Manz, Proceedings Micro. Total Analysis Systems, 2001, p. 349.

${ }^{4}$ J. C. T. Eijkel, H. Stoeri, and A. Manz, Anal. Chem. 72, 2547 (2000).

${ }^{5}$ J. A. Hopwood, J. Microelectromech. Syst. 9, 309 (2000).

${ }^{6}$ S. J. Park, J. Chen, C. Liu, and J. G. Eden, Appl. Phys. Lett. 78, 419 (2001).

${ }^{7}$ C. G. Wilson, A. E. Wendt, and Y. B. Gianchandani, Technical Digest, Solid-State Sensor, Actuator, and Microsystems Workshop, 2002, p. 370.

${ }^{8}$ C. G. Wilson, Ph.D. Thesis, University of Wisconsin-Madison, 2003.

${ }^{9}$ A. L. Ward, J. Appl. Phys. 33, 2789 (1962).

${ }^{10}$ P. Bayle, J. Vacquie, and M. Bayle, Phys. Rev. A 34, 360 (1985).

${ }^{11}$ D. B. Graves and K. F. Jensen, IEEE Trans. Plasma Sci. 14, 78 (1986).
${ }^{12}$ M. Surendra, D. B. Graves, and G. M. Jellum, Phys. Rev. A 41, 1112 (1990).

${ }^{13}$ M. Surendra, D. B. Graves, and L. S. Plano, J. Appl. Phys. 41, 1112 (1990).

${ }^{14}$ J. P. Boeuf, J. Appl. Phys. 63, 1342 (1987).

${ }^{15}$ D. A. Doughty, E. A. Den Hartog, and J. E. Lawler, Phys. Rev. Lett. 58, 2668 (1987).

${ }^{16}$ T. J. Sommerer, J. E. Lawler, and W. N. G. Hitchon, J. Appl. Phys. 64, 1775 (1988).

${ }^{17}$ T. J. Sommerer, W. N. G. Hitchon, and J. E. Lawler, Phys. Rev. A 39, 6356 (1989).

${ }^{18}$ CFD-ACE + User Manual, CFD Research Corporation, Huntsville, AL, 2002.

${ }^{19}$ Yu. B. Golubovskii, V. A. Maiorov, J. Behnke, and J. F. Behnke, Contrib. Papers of HAKONE VII Conference, 2000, Vol. 1, p. 149. 